\title{
Relationship between physician and patient assessment of performance status and survival in a large cohort of patients with haematologic malignancies
}

Michael A Liu ${ }^{1}$, Tammy Hshieh ${ }^{2,3}$, Nolan Condron ${ }^{3}$, Martha Wadleigh ${ }^{3}$, Gregory A Abel ${ }^{3}$ and Jane A Driver $^{\star}, 2,3,4$

${ }^{1}$ University of Arizona College of Medicine, 1501N Campbell Avenue, Tucson, AZ 85724, USA; ${ }^{2}$ Department of Medicine, Division of Aging, Brigham and Women's Hospital, 1620 Tremont Street, Boston, MA 02120, USA; ${ }^{3}$ Department of Medical Oncology, Dana-Farber Cancer Institute, 450 Brookline Avenue, Boston, MA 02215, USA and ${ }^{4}$ Geriatric Research Education and Clinical Center, VA Boston Medical Center, 150 S. Huntington Avenue, Boston, MA 02130, USA

Background: Few studies have investigated the relationship between physician and patient-assessed performance status (PS) in blood cancers.

Methods: Retrospective analysis among 1418 patients with haematologic malignancies seen at Dana-Farber Cancer Institute between 2007 and 2014. We analysed physician-patient agreement of Eastern Cooperative Oncology Group PS using weighted $\kappa$-statistics and survival analysis.

Results: Mean age was 58.6 years and average follow-up was 38 months. Agreement in PS was fair/moderate (weighted $\kappa=0.41$, $95 \% \mathrm{Cl}$ 0.37-0.44). Physicians assigned a better functional status (lower score) than patients (mean 0.60 vs 0.81 ), particularly when patients were young and the disease was aggressive. Both scores independently predicted survival, but physician scores were more accurate. Disagreements in score were associated with poorer survival when physicians rated PS better than patients, and were modified by age, sex and severity of disease.

Conclusions: Physician-patient disagreements in PS score are common and have prognostic significance.

The Eastern Oncology Cooperative Group (ECOG) performance status (PS) provides an overall impression of a patient's level of function in self-care, daily activity and physical activity (Oken et al, 1982). The scale was designed and validated for use by physicians; however, a prospective study of 1115 patients with advanced solid tumours found that patient-assessed PS was strongly predictive of mortality, independent of physician-assessed PS (Loprinzi et al, 1994). A study in another solid-tumour population found that disagreements in ECOG PS between physicians and patients predicted an increased risk of death (Schnadig et al, 2008). In contrast, little is known about differences between patient and physician-assigned PS in blood cancers.

\section{MATERIALS AND METHODS}

Study population and outcomes. We performed a retrospective cohort study of patients with haematologic malignancy presenting to Dana-Farber Cancer Institute (DFCI), a tertiary cancer center in Boston, MA, USA from 2007 to 2014 who participated in a

*Correspondence: Dr JA Driver; E-mail: jdriver@partners.org

Received 26 March 2016; revised 21 July 2016; accepted 26 July 2016; published online 23 August 2016

(c) 2016 Cancer Research UK. All rights reserved 0007-0920/16 
universal research protocol involving a baseline questionnaire in the outpatient setting. The survey included an ECOG PS self-assessment: $(0=$ fully active, able to carry on full activities without restriction; $1=$ restricted in physically strenuous activity, can walk, able to carry out light housework; 2 =ambulatory and capable of all self-care but unable to carry out any work activities, up and about $>50 \%$ of waking hours; 3 = need some help taking care of self; spend more than half day in bed or chair; and $4=$ cannot take care of self at all; spend all day in bed or chair). We abstracted the following variables from the medical record: physician-assigned ECOG PS, age $(<50,50-70$ and $70+$ years), gender, self-reported race/ethnicity, date of initial presentation, date of death or final follow-up, and type of cancer. Haematologic malignancies were categorised as aggressive (diffuse large b-cell lymphoma, mantle cell lymphoma, Hodgkin's lymphoma, Burkitt's lymphoma, acute myeloid leukaemia and acute lymphocytic leukaemia) or non-aggressive (marginal zone lymphoma, follicular lymphoma, chronic myeloid leukaemia, chronic lymphocytic leukaemia, chronic myelomonocytic leukaemia, myelodysplastic syndrome, myeloproliferative neoplasm/myeloproliferative disease and hairy-cell leukaemia). All participants gave written informed consent and the study was approved by the DFCI institutional review board.

Statistical considerations. The level of agreement between patient and physician-assigned PS scores was assessed using weighted $\kappa$-statistics and reported with 95\% confidence intervals (95\% CI). Weighted $\kappa$ takes into account the ordinal scale of the PS observations, such that multiple levels of disagreement are considered. $\kappa$-Statistics were interpreted using benchmarks described by Landis and colleagues (Landis and Koch, 1977). Cox-proportional hazards regression was used to estimate the hazard ratio for survival associated with patient assessment, physician assessments and differences between the two. Patients were followed until they died, were lost to follow-up or reached the end of the study period (December 2014). We compared the predictive power of various models by recording the $-2 \times \log$ likelihood $(-2 \log \mathrm{L})$; models with lower values indicate a more accurate prediction of the outcome. All reported $P$-values were two-sided and a $P$-value of $<0.05$ was considered statistically significant. All analyses were performed using SAS version 9.1.3 (SAS institute, Cary, NC, USA).

\section{RESULTS}

We identified 1418 newly-presenting patients with haematologic malignancies with both patient and physician-assessed ECOG PS. Patients had a mean age of 58.6 years (range 18-97); 95.7\% were white and $58.6 \%$ were male. Mean follow-up was 37.7 months. $40 \%$ had aggressive disease on initial presentation. Thirteen unique physicians provided ECOG assessments.
Overall, patients rated themselves as having a worse PS (mean PS $0.81 ; \pm 0.92$ ) than physicians did (mean PS $0.60 ; \pm 0.73$ ). Physician-patient agreement on PS score was fair to moderate (weighted $\kappa=0.41,95 \%$ CI $0.37-0.44$ ). Agreement increased with patient age from $\kappa=0.34$ for patients younger than 50 years to $\kappa=0.43$ for patients 70 years and older. Weighed $\kappa$-statistics for patient-physician agreement are presented in Table 1.

All physician ECOG scores clearly differentiated distinct survival groups, whereas patient-assessed scores of 1 and 2 did not. After adjustment for age, cancer type and sex, both patient and physician scores were independently associated with overall survival, but the association was stronger for physician scores $(\mathrm{HR}=1.84$; 95\% CI 1.65-2.04 vs $\mathrm{HR}=1.55$; 95\% CI: $1.42-1.69)$. Both scores remained independent predictors of mortality when included together in a multivariable model, and the model that included them both was more accurate in predicting mortality than models with one or the other according to a measure of model fit ( -2 Log L increased from 6802.9 to 6782.9 ). Stratified by age, patient scores of 1 and 2 identified distinct survival groups in those aged $<50$, but not in older patients. In contrast, each level of physician PS predicted a distinct survival risk across all age groups. Stratified by disease type, ECOG score identified distinct survival groups among patients with aggressive malignancies, but in those with indolent disease, scores of 1 and 2 were not statistically different.

Because concordant ratings may be more likely to occur in patients with good PS, we tested for the presence of effect modification by including an interaction term between any disagreement (yes vs no) and physician ECOG PS in the multivariable model. As there was a significant interaction $\left(P_{\text {interaction }}=0.002\right)$, we stratified models of the association between disagreement and survival by physician ECOG scores (Table 2). Patients whose physicians assigned them the best possible PS $(E C O G=0)$ had substantially worse outcomes if they assigned themselves a worse status than the physician, whereas selfassignment of a better functional status than the physician seemed to predict better survival. Stratified analyses (Table 3) showed evidence of effect modification by age, cancer aggressiveness and sex among patients whose physicians rated their PS better than they did; survival was worse if they were younger, male and had an indolent malignancy. Effect modification was less clear in patients whose physicians rated their PS worse than they did.

\section{DISCUSSION}

Our findings suggest that there are systematic differences in the way blood cancer patients and providers report PS, confirming what has been reported for those with solid tumours (Conill et al,

Table 1. ECOG performance status score agreements

\begin{tabular}{|c|c|c|c|c|}
\hline & $\begin{array}{c}\text { Weighted } \\
\kappa \text {-coefficient }\end{array}$ & Agreements, \% (n) & $\begin{array}{c}\text { Physician rates worse than } \\
\text { patient, \% (n) }\end{array}$ & $\begin{array}{c}\text { Physician rates better than } \\
\text { patient, } \%(n)\end{array}$ \\
\hline Overall & $0.41(0.37-0.44)$ & $56.8(806)$ & $13.8(195)$ & $29.4(417)$ \\
\hline \multicolumn{5}{|l|}{ Gender } \\
\hline Female & $0.38(0.33-0.44)$ & 55.0 (323) & $11.8(69)$ & $33.2(195)$ \\
\hline \multicolumn{5}{|l|}{ Age group } \\
\hline Age $70+$ & $0.43(0.36-0.50)$ & $52.4(177)$ & $16.3(55)$ & $31.4(106)$ \\
\hline \multicolumn{5}{|l|}{ Cancer type } \\
\hline Aggressive & $0.37(0.31-0.42)$ & $49.2(272)$ & $11.9(66)$ & $39.0(216)$ \\
\hline Non-aggressive & $0.42(0.37-0.47)$ & 61.7 (534) & 14.9 (129) & $23.3(201)$ \\
\hline
\end{tabular}


Table 2. Physician-patient ECOG PS disagreement and risk of mortality

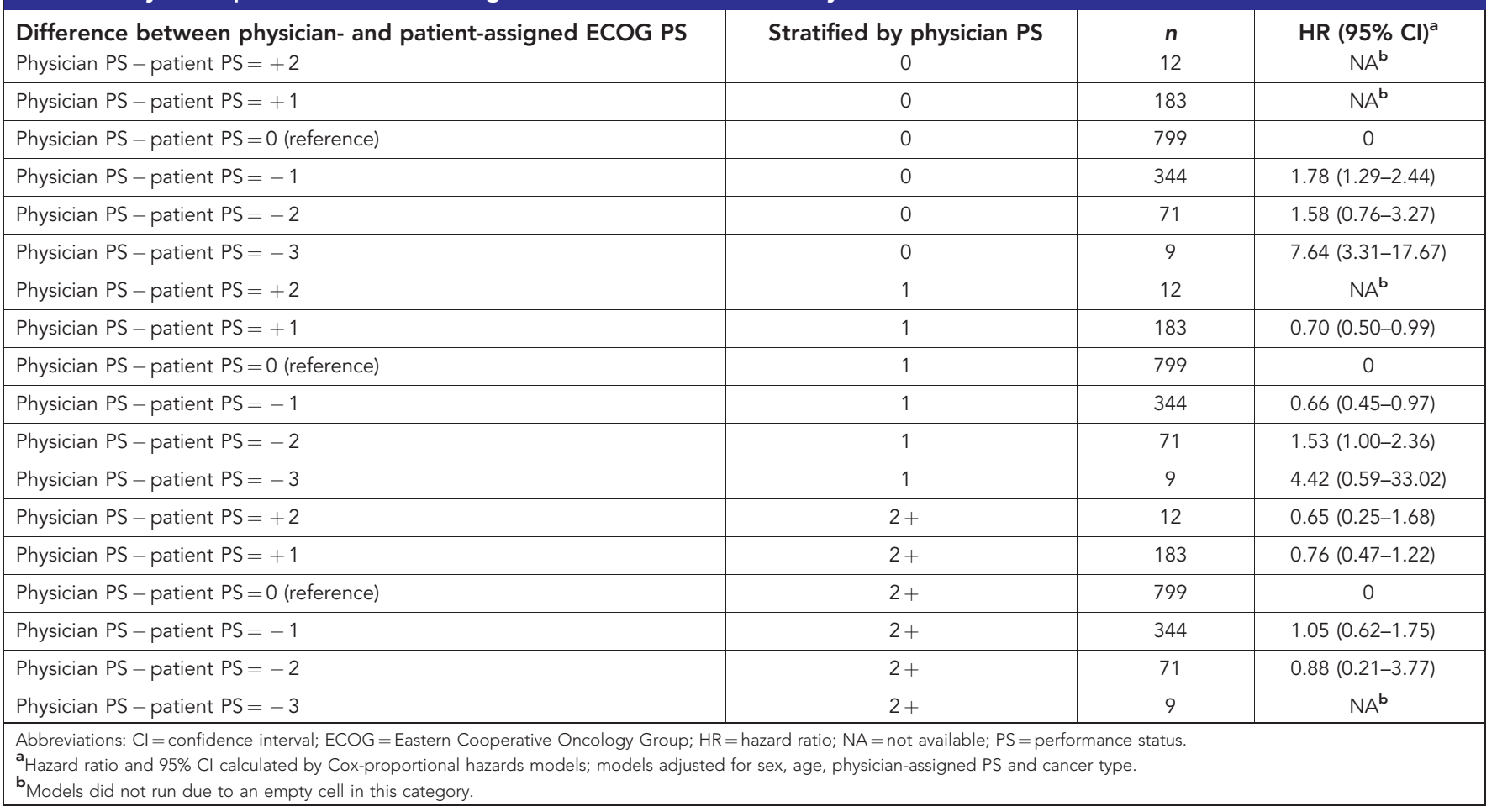

Table 3. Physician-patient ECOG PS disagreement and risk of mortality stratified by age, cancer type and sex

\begin{tabular}{|c|c|c|c|c|}
\hline Stratification variable & $\begin{array}{l}\text { Physician } \\
\text { ECOG score }\end{array}$ & $\mathbf{n}$ & $\begin{array}{l}\text { Physician rates PS better than } \\
\text { patient HR }(95 \% \mathrm{Cl})^{\mathrm{a}}\end{array}$ & $\begin{array}{c}\text { Physician rates PS worse than } \\
\text { patient HR }(95 \% \mathrm{Cl})\end{array}$ \\
\hline Age $\leqslant 60$ years $(n=707)$ & $\begin{array}{c}0 \\
1 \\
2+\end{array}$ & $\begin{array}{c}439 \\
213 \\
55\end{array}$ & $\begin{array}{l}2.71(1.29-3.67) \\
1.60(0.93-2.76) \\
0.96(0.44-2.10)\end{array}$ & $\begin{array}{c}N A^{b} \\
0.83(0.56-1.22) \\
0.66(0.31-1.42)\end{array}$ \\
\hline Age $>60$ years $(n=711)$ & $\begin{array}{c}0 \\
1 \\
2+\end{array}$ & $\begin{array}{l}317 \\
287 \\
107 \\
\end{array}$ & $\begin{array}{l}1.57(1.08-2.28) \\
0.88(0.61-1.28) \\
1.33(0.76-2.33)\end{array}$ & $\begin{array}{c}N A^{\mathbf{b}} \\
0.83(0.56-1.22) \\
0.74(0.45-1.20)\end{array}$ \\
\hline Aggressive cancer $(n=554)$ & $\begin{array}{c}0 \\
1 \\
2+\end{array}$ & $\begin{array}{l}256 \\
224 \\
74\end{array}$ & $\begin{array}{l}1.14(0.71-1.83) \\
0.80(0.54-1.19) \\
0.80(0.58-3.55)\end{array}$ & $\begin{array}{c}N A^{b} \\
0.97(0.61-1.62) \\
0.82(0.45-1.49)\end{array}$ \\
\hline Female sex $(n=587)$ & $\begin{array}{c}0 \\
1 \\
2+ \\
\end{array}$ & $\begin{array}{c}325 \\
202 \\
60 \\
\end{array}$ & $\begin{array}{l}1.32(0.81-2.15) \\
1.35(0.81-2.26) \\
1.09(0.56-2.13)\end{array}$ & $\begin{array}{c}N A^{b} \\
0.83(0.46-1.50) \\
0.44(0.21-0.92)\end{array}$ \\
\hline
\end{tabular}

1990; Ando et al, 2001; Blagden et al, 2003). Agreement was only fair, and we found that greater disagreement conferred higher mortality risk. In older patients, this discordance might reflect a lack of insight due to cognitive impairment, which is a known predictor of poor survival in cancer patients (Extermann and Hurria, 2007). On the other hand, a younger patient who rates his or her function worse than a physician does may have comorbid depression, another potential predictor of poor survival.

Self-reported ECOG PS scores of 1 and 2 did not predict distinct survival categories among older patients. This may reflect the fact that younger patients have a higher baseline level of function, making it easier to perceive a change in status; however, we found that in the subset with aggressive disease, self-reported ECOG of 1 and 2 did identify distinct survival groups in older patients. This may be because aggressive disease results in a more abrupt and noticeable change in functional status than indolent disease. Together, these findings suggest that the traditional ECOG categories may not be adequate for self-report of physical function in older patients, particularly for those with indolent diseases.

In general, we found that a better PS rating by physicians was associated with a worse outcome, and further age- and ECOG PS-stratified analysis revealed that this effect was more prominent 
within the youngest subset of patients and in the subset where physician rated ECOG PS $=0$ (as compared with 1 or $2+$ ). As clinical trials are largely unavailable to patients with poor PS, physicians may tend to assign a better PS than the patient actually has in order that they may qualify for certain treatment regimens (Blagden et al, 2003). However, in our data it was within the non-aggressive cancer group that these differences correlated with the worst mortality outcomes. It is possible that physicians do less detailed investigation of functional status in younger patients and those with non-aggressive disease, and that this unappreciated loss of function is associated with increased mortality.

Strengths of our study include our large and comprehensive clinical cohort of patients with haematologic cancers and longterm follow-up (mean of greater than 3 years). Limitations include the fact that our data are from a single tertiary institution, our limited race-ethnic variability and our inability to understand why patients and physicians had different impressions of PS (i.e., we did not tape-record clinic sessions). We also did not have information on potentially important covariates that could shed light on the reasons for physician-patient discordance, such as patient education level, patient health literacy and performance-status limited clinical trials available at the time of each physician assessment.

In summary, in our cohort of patients with a wide variety of haematologic malignancies, both physician and patient ECOG PS were robust and independent predictors of survival. Although correlated, the two assessments when taken together were better than either alone at predicting mortality. There were substantial differences between physician and patient assessments, and disagreement was also associated with increased mortality risk, particularly among patients with non-aggressive disease. These data suggest a role for both types of assessments in the clinical care of patients with blood cancers.

\section{ACKNOWLEDGEMENTS}

This research was funded by a grant from the MSTAR Program (American Federation for Aging Research/NIH Grant \#T35AG03802705; Michael Liu). TH was supported by Grant No. T32AG000158 from the National Institute on Aging and is supported by a Hartford Center of Excellence Award. GAA and NC are supported by the Mary P.
Murphy Fund for Hematologic Malignancies Research. JAD is supported by a Veterans' Administration Merit Review Award.

\section{CONFLICT OF INTEREST}

The authors declare no conflict of interest.

\section{REFERENCES}

Ando M, Ando Y, Hasegawa Y, Shimokata K, Minami H, Wakai K, Ohno Y, Sakai S (2001) Prognostic value of performance status assessed by patients themselves, nurses, and oncologists in advanced non-small cell lung cancer. Br J Cancer 85(11): 1634.

Blagden S, Charman S, Sharples L, Magee L, Gilligan D (2003) Performance status score: do patients and their oncologists agree? Br J Cancer 89(6): $1022-1027$.

Conill C, Verger E, Salamero M (1990) Performance status assessment in cancer patients. Cancer 65(8): 1864-1866.

Extermann M, Hurria A (2007) Comprehensive geriatric assessment for older patients with cancer. J Clin Oncol 25(14): 1824-1831.

Landis JR, Koch GG (1977) The measurement of observer agreement for categorical data. Biometrics 33(1): 159-174.

Loprinzi CL, Laurie JA, Wieand HS, Krook JE, Novotny PJ, Kugler JW, Bartel J, Law M, Bateman M, Klatt NE (1994) Prospective evaluation of prognostic variables from patient-completed questionnaires. North Central Cancer Treatment Group. J Clin Oncol 12(3): 601-607.

Oken MM, Creech RH, Tormey DC, Horton J, Davis TE, McFadden ET, Carbone PP (1982) Toxicity and response criteria of the Eastern Cooperative Oncology Group. Am J Clin Oncol 5(6): 649-656.

Schnadig ID, Fromme EK, Loprinzi CL, Sloan JA, Mori M, Li H, Beer TM (2008) Patient-physician disagreement regarding performance status is associated with worse survivorship in patients with advanced cancer. Cancer 113(8): 2205-2214.

This work is published under the standard license to publish agreement. After 12 months the work will become freely available and the license terms will switch to a Creative Commons AttributionNonCommercial-Share Alike 4.0 Unported License. 